\title{
Strong convergence theorems of general split equality problems for quasi-nonexpansive mappings
}

Shih-sen Chang ${ }^{1}$ and Ravi P Agarwal ${ }^{*}$

\section{"Correspondence:}

Agarwal@tamuk.edu

2 Department of Mathematics, Texas

A\&M University-Kingsville, 700

University Blvd., Kingsville,

TX 78363-8202, USA

Full list of author information is

available at the end of the article

\begin{abstract}
The purpose of this paper is to introduce and study the general split equality problem and general split equality fixed point problem in the setting of infinite-dimensional Hilbert spaces. Under suitable conditions, we prove that the sequences generated by the proposed new algorithm converges strongly to a solution of the general split equality fixed point problem and the general split equality problem for quasi-nonexpansive mappings in Hilbert spaces. As an application, we shall utilize our results to study the null point problem of maximal monotone operators, the split feasibility problem, and the equality equilibrium problem. The results presented in the paper extend and improve the corresponding results announced by Moudafi et al. (Nonlinear Anal. 79:117-121, 2013; Trans. Math. Program. Appl. 1:1-11, 2013), Eslamian and Latif (Abstr. Appl. Anal. 2013:805104, 2013) and Chen et al. (Fixed Point Theory Appl. 2014:35, 2014), Censor and Elfving (Numer. Algorithms 8:221-239, 1994), Censor and Segal (J. Convex Anal. 16:587-600, 2009) and some others.
\end{abstract}

Keywords: general split equality problem; general split equality fixed point problem; quasi-nonexpansive mapping; split feasibility problem

\section{Introduction}

Let $C$ and $Q$ be nonempty closed convex subsets of real Hilbert spaces $H_{1}$ and $H_{2}$, respectively. The split feasibility problem (SFP) is formulated as

$$
\text { to finding } x^{*} \in C \text { and } A x^{*} \in Q \text {, }
$$

where $A: H_{1} \rightarrow H_{2}$ is a bounded linear operator. In 1994, Censor and Elfving [1] first introduced the SFP in finite-dimensional Hilbert spaces for modeling inverse problems which arise from phase retrievals and in medical image reconstruction [2]. It has been found that the SFP can also be used in various disciplines such as image restoration, and computer tomograph and radiation therapy treatment planning [3-5]. The SFP in an infinitedimensional real Hilbert space can be found in $[2,4,6-10]$.

Assuming that the SFP is consistent, it is not hard to see that $x^{*} \in C$ solves SFP if and only if it solves the fixed point equation

$$
x^{*}=P_{C}\left(I-\gamma A^{*}\left(I-P_{Q}\right) A\right) x^{*},
$$

○2014 Chang and Agarwal; licensee Springer. This is an Open Access article distributed under the terms of the Creative Commons Attribution License (http://creativecommons.org/licenses/by/2.0), which permits unrestricted use, distribution, and reproduction in any medium, provided the original work is properly cited. 
where $P_{C}$ and $P_{Q}$ are the metric projection from $H_{1}$ onto $C$ and from $H_{2}$ onto $Q$, respectively, $\gamma>0$ is a positive constant and $A^{*}$ is the adjoint of $A$.

A popular algorithm to be used to solve SFP (1.1) is due to Byrne's CQ-algorithm [2]:

$$
x_{k+1}=P_{C}\left(I-\gamma A^{*}\left(I-P_{Q}\right) A\right) x_{k}, \quad k \geq 1,
$$

where $\gamma \in(0,2 / \lambda)$ with $\lambda$ being the spectral radius of the operator $A^{*} A$.

Recently, Moudafi [11] introduced the following split equality problem (SEP):

to find $x \in C, y \in Q$ such that $A x=B y$,

where $A: H_{1} \rightarrow H_{3}$ and $B: H_{2} \rightarrow H_{3}$ are two bounded linear operators. Obviously, if $B=I$ (identity mapping on $\mathrm{H}_{2}$ ) and $\mathrm{H}_{3}=\mathrm{H}_{2}$, then (1.2) reduces to (1.1). This kind of split equality problem (1.2) allows asymmetric and partial relations between the variables $x$ and $y$. The interest is to cover many situations, such as decomposition methods for PDEs, applications in game theory, and intensity-modulated radiation therapy.

In order to solve the split equality problem (1.2), Moudafi [11] introduced the following relaxed alternating CQ-algorithm:

$$
\left\{\begin{array}{l}
x_{k+1}=P_{C_{k}}\left(x_{k}-\gamma A^{*}\left(A x_{k}-B y_{k}\right)\right) \\
y_{k+1}=P_{Q_{k}}\left(y_{k}+\beta B^{*}\left(A x_{k}-B y_{k}\right)\right)
\end{array}\right.
$$

where

$$
\begin{array}{ll}
C_{k}=\left\{x \in H_{1} ; c\left(x_{k}\right)+\left\langle\xi_{k}, x-x_{k}\right\rangle \leq 0\right\}, & \xi_{k} \in \partial c\left(x_{k}\right), \\
Q_{k}=\left\{y \in H_{2} ; q\left(y_{k}\right)+\left\langle\eta_{k}, y-y_{k}\right\rangle \leq 0\right\}, & \eta_{k} \in \partial q\left(y_{k}\right),
\end{array}
$$

and $c: H_{1} \rightarrow R$ (respectively $q: H_{2} \rightarrow R$ ) is a convex and subdifferentiable function. Under suitable conditions, he proved that the sequence $\left\{x_{n}\right\}$ defined by (1.4) converges weakly to a solution of the split equality problem (1.2).

Each nonempty closed convex subset of a Hilbert space can be regarded as a set of fixed points of a projection. In [12], Moudafi and Al-Shemas introduced the following split equality fixed point problem:

$$
\text { find } x \in C:=F(S), y \in Q:=F(T) \text { such that } A x=B y \text {, }
$$

where $S: H_{1} \rightarrow H_{1}$ and $T: H_{2} \rightarrow H_{2}$ are two firmly quasi-nonexpansive mappings, $F(S)$ and $F(T)$ denote the fixed point sets of $S$ and $T$, respectively.

To solve the split equality fixed point problem (1.5) for firmly quasi-nonexpansive mappings, Moudafi et al. [11-13] proposed the following iteration algorithm:

$$
\left\{\begin{array}{l}
x_{k+1}=S\left(x_{k}-\gamma_{k} A^{*}\left(A x_{k}-B y_{k}\right)\right), \\
y_{k+1}=T\left(y_{k}+\gamma_{k} B^{*}\left(A x_{k}-B y_{k}\right)\right) .
\end{array}\right.
$$

Very recently, Eslamian and Latif [14] and Chen et al. [15] introduced and studied some kinds of general split feasibility problem and split equality problem in real Hilbert spaces, and under suitable conditions some strong convergence theorems are proved. 
Motivated by the above works, the purpose of this paper is to introduce the following general split equality fixed point problem:

(GSEFP) to find $x \in C:=\bigcap_{i=1}^{\infty} F\left(S_{i}\right), y \in Q:=\bigcap_{i=1}^{\infty} F\left(T_{i}\right)$ such that $A x=B y$,

and the general split equality problem:

(GSEP) to find $x \in C y \in Q$ such that $A x=B y$.

For solving the GSEFP (1.7) and GSEP (1.8), in Sections 3 and 4, we propose an algorithm for finding the solutions of the general split equality fixed point problem and general split equality problem in a Hilbert space. We establish the strong convergence of the proposed algorithms to a solution of GSEFP and GSEP. As applications, in Section 5 we utilize our results to study the split feasibility problem, the null point problem of maximal monotone operators, and the equality equilibrium problem.

\section{Preliminaries}

Let $H$ be a real Hilbert space and $C$ be a nonempty closed convex subset of $H$. In the sequel, denote by $F(T)$ the set of fixed points of a mapping $T$ and by $x_{n} \rightarrow x^{*}$ and $x_{n} \rightarrow x^{*}$, the strong convergence and weak convergence of a sequence $\left\{x_{n}\right\}$ to a point $x^{*}$, respectively.

Recall that a mapping $T: H \rightarrow H$ is said to be nonexpansive, if $\|T x-T y\| \leq\|x-y\|$, $\forall x, y \in H$. A typical example of a nonexpansive mapping is the metric projection $P_{C}$ from $H$ onto $C \subseteq H$ defined by $\left\|x-P_{C} x\right\|=\inf _{y \in C}\|x-y\|$. The metric projection $P_{C}$ is firmly nonexpansive, i.e.,

$$
\left\|P_{C} x-P_{C} y\right\|^{2} \leq\left\langle x-y, P_{C} x-P_{C} y\right\rangle, \quad \forall x, y \in H
$$

and it can be characterized by the fact that

$$
P_{C}(x) \in C \quad \text { and } \quad\left\langle y-P_{C}(x), x-P_{C}(x)\right\rangle \leq 0, \quad \forall x \in H, y \in C .
$$

Definition 2.1 A mapping $T: H \rightarrow H$ is said to be quasi-nonexpansive, if $F(T) \neq \emptyset$, and

$$
\|T x-p\| \leq\|x-p\| \quad \text { for each } x \in H \text { and } p \in F(T)
$$

Lemma 2.2 [16] Let $H$ be a real Hilbert space, and $\left\{x_{n}\right\}$ be a sequence in $H$. Then, for any given sequence $\left\{\lambda_{n}\right\}$ of positive numbers with $\sum_{i=1}^{\infty} \lambda_{n}=1$ such that for any positive integers $i, j$ with $i<j$, the following holds:

$$
\left\|\sum_{i=1}^{\infty} \lambda_{n} x_{n}\right\|^{2} \leq \sum_{i=1}^{\infty} \lambda_{n}\left\|x_{n}\right\|^{2}-\lambda_{i} \lambda_{j}\left\|x_{i}-x_{j}\right\|^{2} .
$$

Lemma 2.3 [17] Let $H$ be a real Hilbert space. For any $x, y \in H$, the following inequality holds:

$$
\|x+y\|^{2} \leq\|x\|^{2}+2\langle y, x+y\rangle .
$$


Lemma 2.4 [18] Let $\left\{t_{n}\right\}$ be a sequence of real numbers. If there exists a subsequence $\left\{n_{i}\right\}$ of $\{n\}$ such that $t_{n_{i}}<t_{n_{i}+1}$ for all $i \geq 1$, then there exists a nondecreasing sequence $\{\tau(n)\}$ with $\tau(n) \rightarrow \infty$ such that for all (sufficiently large) positive integer numbers $n$, the following holds:

$$
t_{\tau(n)} \leq t_{\tau(n)+1}, \quad t_{n} \leq t_{\tau(n)+1} .
$$

In fact,

$$
\tau(n)=\max \left\{k \leq n: t_{k} \leq t_{k+1}\right\}
$$

Definition 2.5 (Demiclosedness principle) Let $C$ be a nonempty closed convex subset of a real Hilbert space $H$, and $T: C \rightarrow C$ be a mapping with $F(T) \neq \emptyset$. Then $I-T$ is said to be demi-closed at zero, if for any sequence $\left\{x_{n}\right\} \subset C$ with $x_{n} \rightarrow x$ and $\left\|x_{n}-T x_{n}\right\| \rightarrow 0$, then $x=T x$

Remark 2.6 It is well known that if $T: C \rightarrow C$ is a nonexpansive mapping, then $I-T$ is demi-closed at zero.

Lemma 2.7 Let $\left\{a_{n}\right\},\left\{b_{n}\right\}$, and $\left\{c_{n}\right\}$ be sequences of positive real numbers satisfying $a_{n+1} \leq$ $\left(1-b_{n}\right) a_{n}+c_{n}$ for all $n \geq 1$. If the following conditions are satisfied:

(1) $b_{n} \in(0,1)$ and $\sum_{n=1}^{\infty} b_{n}=\infty$,

(2) $\sum_{n=1}^{\infty} c_{n}<\infty$, or $\lim \sup _{n \rightarrow \infty} \frac{c_{n}}{b_{n}} \leq 0$,

then $\lim _{n \rightarrow \infty} a_{n}=0$.

\section{Strong convergence theorem for general split equality fixed point problem}

Throughout this section we always assume that

(1) $H_{1}, H_{2}, H_{3}$ are real Hilbert spaces;

(2) $\left\{S_{i}\right\}_{i=1}^{\infty}: H_{1} \rightarrow H_{1}$ and $\left\{T_{i}\right\}_{i=1}^{\infty}: H_{2} \rightarrow H_{2}$ are two families of one-to-one and quasi-nonexpansive mappings;

(3) $A: H_{1} \rightarrow H_{3}$ and $B: H_{2} \rightarrow H_{3}$ are two bounded linear operators;

(4) $f=\left[\begin{array}{l}f_{1} \\ f_{2}\end{array}\right]$, where $f_{i}, i=1,2$ is a $k$-contractive mapping on $H_{i}$ with $k \in(0,1)$;

(5) $C:=\bigcap_{i=1}^{\infty} F\left(S_{i}\right), Q:=\bigcap_{i=1}^{\infty} F\left(T_{i}\right), \Gamma$ is the set of solutions of $\operatorname{GSEFP}(1.7)$,

$$
P=\left[\begin{array}{l}
P_{C} \\
P_{Q}
\end{array}\right], \quad K_{i}=\left[\begin{array}{l}
S_{i} \\
T_{i}
\end{array}\right], \quad G=\left[\begin{array}{ll}
A & -B
\end{array}\right], \quad G^{*} G=\left[\begin{array}{cc}
A^{*} A & -A^{*} B \\
-B^{*} A & B^{*} B
\end{array}\right] ;
$$

(6) for any given $w_{0} \in H_{1} \times H_{2}$, the iterative sequence $\left\{w_{n}\right\} \subset H_{1} \times H_{2}$ is generated by

$$
w_{n+1}=P\left[\alpha_{n} w_{n}+\beta_{n} f\left(w_{n}\right)+\sum_{i=1}^{\infty} \gamma_{n, i}\left(K_{i}\left(I-\lambda_{n, i} G^{*} G\right) w_{n}\right)\right], \quad n \geq 0,
$$

where $\left\{\alpha_{n}\right\},\left\{\beta_{n}\right\},\left\{\gamma_{n, i}\right\}$ are the sequences of nonnegative numbers with

$$
\alpha_{n}+\beta_{n}+\sum_{i=1}^{\infty} \gamma_{n, i}=1 \quad \text { for each } n \geq 0
$$


We are now in a position to give the following main result.

Lemma 3.1 Let $w^{*}=\left(x^{*}, y^{*}\right)$ be a point in $C \times Q$, i.e., $x^{*} \in C=\bigcap_{i=1}^{\infty} F\left(S_{i}\right)$ and $y^{*} \in Q=$ $\bigcap_{i=1}^{\infty} F\left(T_{i}\right)$. Then the following statements are equivalent:

(i) $w^{*}$ is a solution to GSEFP (1.7);

(ii) $w^{*}=K_{i}\left(w^{*}\right)$ for each $i \geq 1$ and $G\left(w^{*}\right)=0$;

(iii) for each $i \geq 1$ and for each $\lambda>0, w^{*}$ solves the fixed point equations:

$$
w^{*}=K_{i} w^{*} \text { and } w^{*}=K_{i}\left(I-\lambda G^{*} G\right) w^{*} .
$$

Proof (i) $\Rightarrow$ (ii). If $w^{*} \in C \times Q$ is a solution to GSEFP (1.7), then for each $i \geq 1, w^{*}=K_{i} w^{*}$, and $A x^{*}=B y^{*}$. This implies that for each $i \geq 1, w^{*}=K_{i} w^{*}$, and

$$
G\left(w^{*}\right)=\left[\begin{array}{ll}
A & -B
\end{array}\right]\left[\begin{array}{l}
x^{*} \\
y^{*}
\end{array}\right]=A x^{*}-B y^{*}=0 .
$$

(ii) $\Rightarrow$ (iii). If $w^{*}=K_{i}\left(w^{*}\right), \forall i \geq 1$ and $G\left(w^{*}\right)=0$, it is easy to see that (3.2) holds.

(iii) $\Rightarrow$ (i). From (3.2), for each $i \geq 1$ we have $K_{i} w^{*}=K_{i}\left(I-\lambda G^{*} G\right) w^{*}$. Since $S_{i}$ and $T_{i}$ both are one-to-one, so is $K_{i}$. Hence we have $\left\|w^{*}-\left(I-\lambda G^{*} G\right) w^{*}\right\|=0$, for any $\lambda>0$. This implies that $G^{*} G\left(w^{*}\right)=0$, and so

$$
0=\left\langle G^{*} G w^{*}, w^{*}\right\rangle=\left\langle G w^{*}, G w^{*}\right\rangle=\left\|G w^{*}\right\|^{2},
$$

i.e., $G\left(w^{*}\right)=A x^{*}-B y^{*}=0$.

This completes the proof of Lemma 3.1.

Lemma 3.2 If $\lambda \in\left(0, \frac{2}{L}\right)$, where $L=\|G\|^{2}$, then $\left(I-\lambda G^{*} G\right): H_{1} \times H_{2} \rightarrow H_{1} \times H_{2}$ is a nonexpansive mapping.

Proof In fact for any $w, u \in H_{1} \times H_{2}$, we have

$$
\begin{aligned}
\| & \left(I-\lambda G^{*} G\right) u-\left(I-\lambda G^{*} G\right) w \|^{2} \\
& =\left\|(u-w)-\lambda G^{*} G(u-w)\right\|^{2} \\
& =\|u-w\|^{2}+\lambda^{2}\left\|G^{*} G(u-w)\right\|^{2}-2 \lambda\left\langle u-w, G^{*} G(u-w)\right\rangle \\
& \leq\|u-w\|^{2}+\lambda^{2} L\|G(u-w)\|^{2}-2 \lambda\langle G(u-w), G(u-w)\rangle \\
& =\|u-w\|^{2}+\lambda^{2} L\|G(u-w)\|^{2}-2 \lambda\|G(u-w)\|^{2} \\
& =\|u-w\|^{2}-\lambda(2-\lambda L)\|G(u-w)\|^{2} \\
& \leq\|u-w\|^{2} .
\end{aligned}
$$

This completes the proof.

Theorem 3.3 Let $H_{1}, H_{2}, H_{3},\left\{S_{i}\right\},\left\{T_{i}\right\}, A, B, f, C, Q, \Gamma, P, G, K_{i}, G^{*} G$ satisfy the above conditions (1)-(5). Let $\left\{w_{n}\right\}$ be the sequence defined by (3.1). If the solution set $\Gamma$ of GSEFP (1.7) is nonempty and the following conditions are satisfied: 
(i) $\alpha_{n}+\beta_{n}+\sum_{i=1}^{\infty} \gamma_{n, i}=1$, for each $n \geq 0$;

(ii) $\lim _{n \rightarrow \infty} \beta_{n}=0$, and $\sum_{n=0}^{\infty} \beta_{n}=\infty$;

(iii) $\liminf _{n \rightarrow \infty} \alpha_{n} \gamma_{n, i}>0$ for each $i \geq 1$;

(iv) $\left\{\lambda_{n, i}\right\} \subset\left(0, \frac{2}{L}\right)$ for each $i \geq 1$, where $L=\|G\|^{2}$;

(v) for each $i \geq 1$, the mapping $I-K_{i}\left(I-\lambda_{n, i} G^{*} G\right)$ is demi-closed at zero,

then the sequence $\left\{w_{n}\right\}$ converges strongly to $w^{*}=P_{\Gamma} f\left(w^{*}\right)$ which is a solution of GSEFP (1.7).

Proof (I) First we prove that the sequence $\left\{w_{n}\right\}$ is bounded.

In fact, for any given $z \in \Gamma$, it follows from Lemma 3.1 that

$$
G(z)=0, \quad K_{i} z=z \quad \text { and } \quad z=K_{i}\left(I-\lambda_{n, i} G^{*} G\right) z \quad \text { for each } i \geq 1 .
$$

By the assumptions and Lemma 3.2, for each $\lambda \in\left(0, \frac{2}{L}\right),\left(I-\lambda G^{*} G\right): H_{1} \times H_{2} \rightarrow H_{1} \times H_{2}$ is nonexpansive, and for each $i \geq 1, K_{i}=\left[\begin{array}{c}S_{i} \\ T_{i}\end{array}\right]$ is quasi-nonexpansive, hence we have

$$
\begin{aligned}
\left\|w_{n+1}-z\right\| & =\left\|P\left[\alpha_{n} w_{n}+\beta_{n} f\left(w_{n}\right)+\sum_{i=1}^{\infty} \gamma_{n, i} K_{i}\left(I-\lambda_{n, i} G^{*} G\right) w_{n}\right]-P(z)\right\| \\
& \leq \alpha_{n}\left\|w_{n}-z\right\|+\beta_{n}\left\|f\left(w_{n}\right)-z\right\|+\sum_{i=1}^{\infty} \gamma_{n, i}\left\|K_{i}\left(I-\lambda_{n, i} G^{*} G\right) w_{n}-z\right\| \\
& \leq \alpha_{n}\left\|w_{n}-z\right\|+\beta_{n}\left\|f\left(w_{n}\right)-z\right\|+\sum_{i=1}^{\infty} \gamma_{n, i}\left\|\left(I-\lambda_{n, i} G^{*} G\right) w_{n}-z\right\| \\
& \leq \alpha_{n}\left\|w_{n}-z\right\|+\beta_{n}\left\|f\left(w_{n}\right)-z\right\|+\sum_{i=1}^{\infty} \gamma_{n, i}\left\|\left(I-\lambda_{n, i} G^{*} G\right) w_{n}-\left(I-\lambda_{n, i} G^{*} G\right) z\right\| \\
& \leq \alpha_{n}\left\|w_{n}-z\right\|+\beta_{n}\left\|f\left(w_{n}\right)-z\right\|+\sum_{i=1}^{\infty} \gamma_{n, i}\left\|w_{n}-z\right\| \\
& =\left(1-\beta_{n}\right)\left\|w_{n}-z\right\|+\beta_{n}\left\|f\left(w_{n}\right)-z\right\| \\
& \leq\left(1-\beta_{n}\right)\left\|w_{n}-z\right\|+\beta_{n}\left\|f\left(w_{n}\right)-f(z)\right\|+\beta_{n}\|f(z)-z\| \\
& \leq\left(1-\beta_{n}\right)\left\|w_{n}-z\right\|+k \beta_{n}\left\|w_{n}-z\right\|+\beta_{n}\|f(z)-z\| \\
& =\left(1-(1-k) \beta_{n}\right)\left\|w_{n}-z\right\|+(1-k) \beta_{n} \frac{1}{1-k}\|f(z)-z\| \\
& \leq \max \left\{\left\|w_{n}-z\right\|, \frac{1}{1-k}\|f(z)-z\|\right\} .
\end{aligned}
$$

By induction, we can prove that

$$
\left\|w_{n}-z\right\| \leq \max \left\{\left\|w_{0}-z\right\|, \frac{1}{1-k}\|f(z)-z\|\right\} .
$$

This shows that $\left\{w_{n}\right\}$ is bounded, and so is $\left\{f\left(w_{n}\right)\right\}$.

(II) Now we prove that the following inequality holds:

$$
\begin{aligned}
& \alpha_{n} \gamma_{n, i}\left\|w_{n}-K_{i}\left(I-\lambda_{n, i} G^{*} G\right) w_{n}\right\|^{2} \\
& \quad \leq\left\|w_{n}-z\right\|^{2}-\left\|w_{n+1}-z\right\|^{2}+\beta_{n}\left\|f\left(w_{n}\right)-z\right\|^{2} \quad \text { for each } i \geq 1 .
\end{aligned}
$$


Indeed, it follows from (3.1) and Lemma 2.2 that for each $i \geq 1$

$$
\begin{aligned}
\left\|w_{n+1}-z\right\|^{2}= & \left\|P\left[\alpha_{n}\left(w_{n}-z\right)+\beta_{n}\left(f\left(w_{n}\right)-z\right)+\sum_{i=1}^{\infty} \gamma_{n, i}\left(K_{i}\left(I-\lambda_{n, i} G^{*} G\right) w_{n}\right)\right]-z\right\|^{2} \\
\leq & \alpha_{n}\left\|w_{n}-z\right\|^{2}+\beta_{n}\left\|f\left(w_{n}\right)-z\right\|^{2}+\sum_{i=1}^{\infty} \gamma_{n, i}\left\|K_{i}\left(I-\lambda_{n, i} G^{*} G\right) w_{n}-z\right\|^{2} \\
& -\alpha_{n} \gamma_{n, i}\left\|w_{n}-K_{i}\left(I-\lambda_{n, i} G^{*} G\right) w_{n}\right\|^{2} \\
\leq & \alpha_{n}\left\|w_{n}-z\right\|^{2}+\beta_{n}\left\|f\left(w_{n}\right)-z\right\|^{2}+\sum_{i=1}^{\infty} \gamma_{n, i}\left\|w_{n}-z\right\|^{2} \\
& -\alpha_{n} \gamma_{n, i}\left\|w_{n}-K_{i}\left(I-\lambda_{n, i} G^{*} G\right) w_{n}\right\|^{2} \\
= & \left(1-\beta_{n}\right)\left\|w_{n}-z\right\|^{2}+\beta_{n}\left\|f\left(w_{n}\right)-z\right\|^{2} \\
& -\alpha_{n} \gamma_{n, i}\left\|w_{n}-K_{i}\left(I-\lambda_{n, i} G^{*} G\right) w_{n}\right\|^{2} .
\end{aligned}
$$

This implies that for each $i \geq 1$

$$
\alpha_{n} \gamma_{n, i}\left\|w_{n}-K_{i}\left(I-\lambda_{n, i} G^{*} G\right) w_{n}\right\|^{2} \leq\left\|w_{n}-z\right\|^{2}-\left\|w_{n+1}-z\right\|^{2}+\beta_{n}\left\|f\left(w_{n}\right)-z\right\|^{2}
$$

Inequality (3.3) is proved.

It is easy to see that the solution set $\Gamma$ of GSEFP (1.7) is a nonempty closed and convex subset in $C \times Q$, hence the metric projection $P_{\Gamma}$ is well defined. In addition, since $P_{\Gamma} f$ : $H_{1} \times H_{2} \rightarrow H_{1} \times H_{2}$ is a contractive mapping, there exists a $w^{*} \in \Gamma$ such that

$$
w^{*}=P_{\Gamma} f\left(w^{*}\right) .
$$

(III) Now we prove that $w_{n} \rightarrow w^{*}$.

For this purpose, we consider two cases.

Case I. Suppose that the sequence $\left\{\left\|w_{n}-w^{*}\right\|\right\}$ is monotone. Since $\left\{\left\|w_{n}-w^{*}\right\|\right\}$ is bounded, $\left\{\left\|w_{n}-w^{*}\right\|\right\}$ is convergent. Since $w^{*} \in \Gamma$, in (3.3) taking $z=w^{*}$ and letting $n \rightarrow \infty$, in view of conditions (ii) and (iii), we have

$$
\lim _{n \rightarrow \infty}\left\|w_{n}-K_{i}\left(I-\lambda_{n, i} G^{*} G\right) w_{n}\right\|=0 \quad \text { for each } i \geq 1
$$

On the other hand, by Lemma 2.3 and (3.1), we have

$$
\begin{aligned}
\left\|w_{n+1}-w^{*}\right\|^{2}= & \left\|P\left[\alpha_{n} w_{n}+\beta_{n} f\left(w_{n}\right)+\sum_{i=1}^{\infty} \gamma_{n, i} K_{i}\left(I-\lambda_{n, i} G^{*} G\right) w_{n}\right]-w^{*}\right\|^{2} \\
\leq & \| \alpha_{n}\left(w_{n}-w^{*}\right)+\beta_{n}\left(f\left(w_{n}\right)-w^{*}\right) \\
& +\sum_{i=1}^{\infty} \gamma_{n, i}\left(K_{i}\left(I-\lambda_{n, i} G^{*} G\right) w_{n}-w^{*}\right) \|^{2} \\
\leq & \left\|\alpha_{n}\left(w_{n}-w^{*}\right)+\sum_{i=1}^{\infty} \gamma_{n, i}\left(K_{i}\left(I-\lambda_{n, i} G^{*} G\right) w_{n}-w^{*}\right)\right\|^{2}
\end{aligned}
$$




$$
\begin{aligned}
& +2 \beta_{n}\left\langle f\left(w_{n}\right)-w^{*}, w_{n+1}-w^{*}\right\rangle \quad \text { (by Lemma 2.3) } \\
\leq & \left\{\alpha_{n}\left\|w_{n}-w^{*}\right\|+\sum_{i=1}^{\infty} \gamma_{n, i}\left\|w_{n}-w^{*}\right\|\right\}^{2} \\
& +2 \beta_{n}\left\langle f\left(w_{n}\right)-f\left(w^{*}\right), w_{n+1}-w^{*}\right\rangle+2 \beta_{n}\left\langle f\left(w^{*}\right)-w^{*}, w_{n+1}-w^{*}\right\rangle \\
= & \left(1-\beta_{n}\right)^{2}\left\|w_{n}-w^{*}\right\|^{2}+2 \beta_{n} k\left\|w_{n}-w^{*}\right\|\left\|w_{n+1}-w^{*}\right\| \\
& +2 \beta_{n}\left\langle f\left(w^{*}\right)-w^{*}, w_{n+1}-w^{*}\right\rangle \\
\leq & \left(1-\beta_{n}\right)^{2}\left\|w_{n}-w^{*}\right\|^{2}+\beta_{n} k\left\{\left\|w_{n}-w^{*}\right\|^{2}+\left\|w_{n+1}-w^{*}\right\|^{2}\right\} \\
& +2 \beta_{n}\left\langle f\left(w^{*}\right)-w^{*}, w_{n+1}-w^{*}\right\rangle .
\end{aligned}
$$

Simplifying we have

$$
\begin{aligned}
\left\|w_{n+1}-w^{*}\right\|^{2} \leq & \frac{\left(1-\beta_{n}\right)^{2}+\beta_{n} k}{1-\beta_{n} k}\left\|w_{n}-w^{*}\right\|^{2}+\frac{2 \beta_{n}}{1-\beta_{n} k}\left\langle f\left(w^{*}\right)-w^{*}, w_{n+1}-w^{*}\right\rangle \\
= & \frac{1-2 \beta_{n}+\beta_{n} k}{1-\beta_{n} k}\left\|w_{n}-w^{*}\right\|^{2}+\frac{\beta_{n}^{2}}{1-\beta_{n} k}\left\|w_{n}-w^{*}\right\|^{2} \\
& +\frac{2 \beta_{n}}{1-\beta_{n} k}\left\langle f\left(w^{*}\right)-w^{*}, w_{n+1}-w^{*}\right\rangle \\
= & \left(1-\frac{2(1-k) \beta_{n}}{1-\beta_{n} k}\right)\left\|w_{n}-w^{*}\right\|^{2} \\
& +\frac{2(1-k) \beta_{n}}{1-\beta_{n} k}\left\{\frac{\beta_{n} M}{2(1-k)}+\frac{1}{1-k}\left\langle f\left(w^{*}\right)-w^{*}, w_{n+1}-w^{*}\right\rangle\right\} \\
= & \left(1-\eta_{n}\right)\left\|w_{n}-w^{*}\right\|^{2}+\eta_{n} \delta_{n},
\end{aligned}
$$

where $\eta_{n}=\frac{2(1-k) \beta_{n}}{1-\beta_{n} k}, \delta_{n}=\frac{\beta_{n} M}{2(1-k)}+\frac{1}{1-k}\left\langle f\left(w^{*}\right)-w^{*}, w_{n+1}-w^{*}\right\rangle, M:=\sup _{n \geq 0}\left\|w_{n}-w^{*}\right\|$.

By condition (ii), $\lim _{n \rightarrow \infty} \beta_{n}=0$ and $\sum_{n=1}^{\infty} \beta_{n}=\infty$, and so $\sum_{n=1}^{\infty} \eta_{n}=\infty$.

Next we prove that

$$
\limsup _{n \rightarrow \infty} \delta_{n} \leq 0
$$

In fact, since $\left\{w_{n}\right\}$ is bounded in $C \times Q$, there exists a subsequence $\left\{w_{n_{k}}\right\} \subset\left\{w_{n}\right\}$ with $w_{n_{k}} \rightarrow v^{*}$ (some point in $\left.C \times Q\right)$, and $\lambda_{n_{k}, i} \rightarrow \lambda_{i} \in\left(0, \frac{2}{L}\right)$ such that

$$
\lim _{n \rightarrow \infty}\left\langle f\left(w^{*}\right)-w^{*}, w_{n_{k}}-w^{*}\right\rangle=\limsup _{n \rightarrow \infty}\left\langle f\left(w^{*}\right)-w^{*}, w_{n}-w^{*}\right\rangle .
$$

In view of (3.5)

$$
\left\|w_{n_{k}}-K_{i}\left(I-\lambda_{n_{k}, i} G^{*} G\right) w_{n_{k}}\right\| \rightarrow 0 \quad \text { for each } i \geq 1
$$

Again by the assumption that for each $i \geq 1$, the mapping $I-K_{i}\left(I-\lambda_{n, i} G^{*} G\right)$ is demi-closed at zero, hence we have

$$
v^{*}=K_{i} v^{*} \quad \text { and } \quad v^{*}=K_{i}\left(I-\lambda_{n, i} G^{*} G\right) v^{*}, \quad \forall i \geq 1 .
$$


By Lemma 3.1, this implies that $v^{*} \in \Gamma$. In addition, since $w^{*}=P_{\Gamma} f\left(w^{*}\right)$, we have

$$
\begin{aligned}
\limsup _{n \rightarrow \infty}\left\langle f\left(w^{*}\right)-w^{*}, w_{n}-w^{*}\right\rangle & =\lim _{n \rightarrow \infty}\left\langle f\left(w^{*}\right)-w^{*}, w_{n_{k}}-w^{*}\right\rangle \\
& =\left\langle f\left(w^{*}\right)-w^{*}, v^{*}-w^{*}\right\rangle \leq 0 .
\end{aligned}
$$

This shows that (3.7) is true. Taking $a_{n}=\left\|w_{n}-w^{*}\right\|^{2}, b_{n}=\eta_{n}$, and $c_{n}=\delta_{n} \eta_{n}$ in Lemma 2.7, all conditions in Lemma 2.7 are satisfied. We have $w_{n} \rightarrow w^{*}$.

Case II. If the sequence $\left\{\left\|w_{n}-w^{*}\right\|\right\}$ is not monotone, by Lemma 2.4, there exists a sequence of positive integers: $\{\tau(n)\}, n \geq n_{0}$ (where $n_{0}$ is large enough) such that

$$
\tau(n)=\max \left\{k \leq n:\left\|w_{k}-w^{*}\right\| \leq\left\|w_{k+1}-w^{*}\right\|\right\} .
$$

Clearly $\{\tau(n)\}$ is nondecreasing, $\tau(n) \rightarrow \infty$ as $n \rightarrow \infty$, and for all $n \geq n_{0}$

$$
\left\|w_{\tau(n)}-w^{*}\right\| \leq\left\|w_{\tau(n)+1}-w^{*}\right\| ; \quad\left\|w_{n}-w^{*}\right\| \leq\left\|w_{\tau(n)+1}-w^{*}\right\|
$$

Therefore $\left\{\left\|w_{\tau(n)}-w^{*}\right\|\right\}$ is a nondecreasing sequence. According to Case I, $\lim _{n \rightarrow \infty} \| w_{\tau(n)}-$ $w^{*} \|=0$ and $\lim _{n \rightarrow \infty}\left\|w_{\tau(n)+1}-w^{*}\right\|=0$. Hence we have

$$
0 \leq\left\|w_{n}-w^{*}\right\| \leq \max \left\{\left\|w_{n}-w^{*}\right\|,\left\|w_{\tau(n)}-w^{*}\right\|\right\} \leq\left\|w_{\tau(n)+1}-w^{*}\right\| \rightarrow 0, \quad \text { as } n \rightarrow \infty
$$

This implies that $w_{n} \rightarrow w^{*}$ and $w^{*}=P_{\Gamma} f\left(w^{*}\right)$ is a solution of GSEFP (1.7).

This completes the proof of Theorem 3.3.

Remark 3.4 Theorem 3.3 extends and improves the main results in Moudafi et al. [11-13] in the following aspects:

(a) For the mappings, we extend the mappings from firmly quasi-nonexpansive mappings to an infinite family of one-to-one quasi-nonexpansive mappings.

(b) For the algorithms, we propose new iterative algorithms which are different from ones given in [11-13].

(c) For the convergence, the iterative sequence proposed by our algorithm converges strongly to a solution of GSEFP (1.7). But the iterative sequences proposed in [11-13] are only of weak convergence to a solution of the split equality problem.

\section{Strong convergence theorem for general split equality problem}

Throughout this section we always assume that

(1) $H_{1}, H_{2}, H_{3}$ are real Hilbert spaces; $\left\{C_{i}\right\}_{i=1}^{\infty} \subset H_{1}$ and $\left\{Q_{i}\right\}_{i=1}^{\infty} \subset H_{2}$ are two families of nonempty closed and convex subsets with $C=\bigcap_{i=1}^{\infty} C_{i} \neq \emptyset$ and $Q=\bigcap_{i=1}^{\infty} Q_{i} \neq \emptyset$;

(2) $P_{C_{i}}$ (resp. $P_{Q_{i}}$ ) is the metric projection from $H_{1}$ onto $C_{i}$ (resp. $H_{2}$ onto $Q_{i}$ ), and $P_{i}:=\left[\begin{array}{l}P_{C_{i}} \\ P_{Q_{i}}\end{array}\right], i=1,2, \ldots$, and $P:=\left[\begin{array}{l}P_{C} \\ P_{Q}\end{array}\right]$;

(3) $A: H_{1} \rightarrow H_{3}$ and $B: H_{2} \rightarrow H_{3}$ are two bounded linear operators;

(4) $f, G, G^{*} G$ are the same as in Theorem 3.3.

The so-called general split equality problem (GSEP) is

to find $x \in C y \in Q$ such that $A x=B y$. 
Lemma 4.1 Let $H_{1}, H_{2}, H_{3}, P,\left\{P_{i}\right\}, A, B, f, C, Q, G, G^{*} G$ be the same as above. Then a point $w^{*}=\left(x^{*}, y^{*}\right)$ is a solution to GSEP (4.1), if and only if for each $i \geq 1$ and for each $\lambda>0$, $w^{*}$ solves the following fixed point equations:

$$
w^{*}=P_{i} w^{*} \quad \text { and } \quad w^{*}=P_{i}\left(I-\lambda G^{*} G\right) w^{*} .
$$

Proof In fact, a point $w^{*}=\left(x^{*}, y^{*}\right)$ is a solution of GSEP (4.1)

$$
\begin{aligned}
& \Leftrightarrow \quad w^{*}=\left(x^{*}, y^{*}\right) \in C \times Q \quad \text { and } A x^{*}=B y^{*} \\
& \Leftrightarrow \quad \text { for each } i \geq 1, \quad x^{*}=P_{C_{i}}\left(x^{*}\right), \quad y^{*}=P_{Q_{i}}\left(y^{*}\right) \quad \text { and } \quad A x^{*}=B y^{*} \\
& \Leftrightarrow \quad w^{*}=P_{i}\left(w^{*}\right) \text { and } A x^{*}=B y^{*} \\
& \Leftrightarrow\left\{\begin{array}{l}
A x^{*}=P_{A\left(C_{i}\right) \cap B\left(Q_{i}\right)} B y^{*}, \\
B y^{*}=P_{B\left(Q_{i}\right) \cap A\left(C_{i}\right)} A x^{*}
\end{array}\right. \\
& \Leftrightarrow \begin{cases}\left\langle A x^{*}-P_{B\left(Q_{i}\right)} B y^{*}, A u-A x^{*}\right\rangle \geq 0, & \forall u \in C_{i}, \\
\left\langle B y^{*}-P_{A\left(C_{i}\right)} A x^{*}, B v-B y^{*}\right\rangle \geq 0, & \forall v \in Q_{i}\end{cases} \\
& \Leftrightarrow\left\{\begin{array}{l}
\left\langle A x^{*}-B y^{*}, A u-A x^{*}\right\rangle \geq 0, \quad \forall u \in C_{i}, \\
\left\langle B y^{*}-A x^{*}, B v-B y^{*}\right\rangle \geq 0, \quad \forall v \in Q_{i}
\end{array}\right. \\
& \Leftrightarrow \begin{cases}\left\langle\gamma A^{*}\left(A x^{*}-B y^{*}\right), u-x^{*}\right\rangle \geq 0, & \forall u \in C_{i}, \gamma>0, \\
\left\langle\gamma B^{*}\left(B y^{*}-A x^{*}\right), v-y^{*}\right\rangle \geq 0, & \forall v \in Q_{i}, \gamma>0\end{cases} \\
& \Leftrightarrow\left\{\begin{array}{l}
\left\langle x^{*}-\left(x^{*}-\gamma A^{*}\left(A x^{*}-B y^{*}\right)\right), u-x^{*}\right\rangle \geq 0, \quad \forall u \in C_{i}, \gamma>0, \\
\left\langle y^{*}-\left(y^{*}-\gamma B^{*}\left(B y^{*}-A x^{*}\right)\right), v-y^{*}\right\rangle \geq 0, \quad \forall v \in Q_{i}, \gamma>0
\end{array}\right. \\
& \Leftrightarrow\left\{\begin{array}{l}
x^{*}=P_{C_{i}}\left(x^{*}-\gamma A^{*}\left(A x^{*}-B y^{*}\right)\right), \\
y^{*}=P_{Q_{i}}\left(y^{*}-\gamma B^{*}\left(B y^{*}-A x^{*}\right)\right)
\end{array}\right. \\
& \Leftrightarrow \quad w^{*}=P_{i}\left(I-\gamma G^{*} G\right) w^{*} \quad \text { and } \quad w^{*}=P_{i} w^{*} .
\end{aligned}
$$

This completes the proof of Lemma 4.1.

The metric projections $P_{C_{i}}$ and $P_{Q_{i}}$ are nonexpansive with $F\left(P_{C_{i}}\right)=C_{i}$ and $F\left(P_{Q_{i}}\right)=Q_{i}$, $i \geq 1$. This implies that the metric projections $P_{C_{i}}$ and $P_{Q_{i}}$ all are quasi-nonexpansive. In addition, by Lemma 3.2, for each $i \geq 1$ and each $\lambda \in\left(0, \frac{2}{L}\right)$, the mapping $P_{i}\left(I-\lambda G^{*} G\right)$ : $H_{1} \times H_{2} \rightarrow C_{i} \times Q_{i}$ is nonexpansive. By Remark 2.6, for each $i \geq 1$ and each $\lambda \in\left(0, \frac{2}{L}\right)$, the mapping $\left(I-P_{i}\left(I-\lambda G^{*} G\right)\right)$ is demi-closed at zero.

Consequently, we have the following.

Theorem 4.2 Let $H_{1}, H_{2}, H_{3}, P,\left\{P_{i}\right\}, A, B, f, C, Q, G, G^{*} G$ be the same as above. Let $\left\{w_{n}\right\}$ be the sequence generated by $w_{0} \in H_{1} \times H_{2}$

$$
w_{n+1}=P\left[\alpha_{n} w_{n}+\beta_{n} f\left(w_{n}\right)+\sum_{i=1}^{\infty} \gamma_{n, i} P_{i}\left(I-\lambda_{n, i} G^{*} G\right) w_{n}\right], \quad n \geq 0 .
$$

If the solution set $\Gamma_{1}$ of GSEP (4.1) is nonempty and the following conditions are satisfied:

(i) $\alpha_{n}+\beta_{n}+\sum_{i=1}^{\infty} \gamma_{n, i}=1$, for each $n \geq 0$; 
(ii) $\lim _{n \rightarrow \infty} \beta_{n}=0$, and $\sum_{n=0}^{\infty} \beta_{n}=\infty$;

(iii) $\liminf _{n \rightarrow \infty} \alpha_{n} \gamma_{n, i}>0$ for each $i \geq 1$;

(iv) $\left\{\lambda_{n, i}\right\} \subset\left(0, \frac{2}{L}\right)$ for each $i \geq 1$, where $L=\|G\|^{2}$,

then the sequence $\left\{w_{n}\right\}$ defined by (4.3) converges strongly to a solution $w^{*}$ of GSEP (4.1) and $w^{*}=P_{\Gamma_{1}} f\left(w^{*}\right)$.

Proof Taking $S_{i}=P_{C_{i}}, T_{i}=P_{Q_{i}}$, and $K_{i}=P_{i}, i=1,2, \ldots$ in Theorem 3.3, we know that $S_{i}$ and $T_{i}$ both are nonexpansive with $F\left(S_{i}\right)=C_{i}$ and $F\left(T_{i}\right)=Q_{i}$ and so they are quasinonexpansive mappings, and $C=\bigcap_{i=1}^{\infty} F\left(S_{i}\right)$ and $Q=\bigcap_{i=1}^{\infty} F\left(T_{i}\right)$. Therefore all conditions in Theorem 3.3 are satisfied. The conclusion of Theorem 4.2 can be obtained from Lemma 4.1 and Theorem 3.3 immediately.

Remark 4.3 Theorem 4.2 extends and improves the corresponding results in Censor and Elfving [1], Moudafi et al. [11, 12], Eslamian and Latif [14], Chen et al. [15], Censor and Segal [19].

\section{Applications}

In this section we shall utilize the results presented in the paper to give some applications.

\subsection{Application to split feasibility problem}

Let $C \subset H_{1}$ and $Q \subset H_{2}$ be two nonempty closed convex subsets and $A: H_{1} \rightarrow H_{2}$ be a bounded linear operator. The so-called split feasibility problem (SFP) [1] is to find

$$
x \in C, y \in Q \text { such that } A x=y \text {. }
$$

Let $P_{C}$ and $P_{Q}$ be the metric projection from $H_{1}$ onto $C$ and $H_{2}$ onto $Q$, respectively. Thus $F\left(P_{C}\right)=C$ and $F\left(P_{Q}\right)=Q$. From Theorem 4.2 we have the following.

Theorem 5.1 Let $H_{1}, H_{2}$ be two real Hilbert spaces, $A: H_{1} \rightarrow H_{2}$ be a bounded linear operator and $I$ be the identity mapping on $H_{2}$. Let $C \subset H_{1}$ and $Q \subset H_{2}$ be nonempty closed convex subsets and $P_{C}$ and $P_{Q}$ are the metric projections from $H_{1}$ onto $C$ and $H_{2}$ onto $Q$, respectively. Let $\left\{w_{n}\right\}$ be the sequence generated by $w_{0} \in H_{1} \times H_{2}$ :

$$
w_{n+1}=P\left[\alpha_{n} w_{n}+\beta_{n} f\left(w_{n}\right)+\gamma_{n} P\left(I-\lambda_{n} U^{*} U\right) w_{n}\right], \quad n \geq 0,
$$

where $f$ is the mapping as given in Theorem 4.2 and

$$
U=\left[\begin{array}{ll}
A & -I
\end{array}\right], \quad P=\left[\begin{array}{l}
P_{C} \\
P_{Q}
\end{array}\right], \quad U^{*} U=\left[\begin{array}{cc}
A^{*} A & -A^{*} \\
-A & I
\end{array}\right] .
$$

If the solution set $\Gamma_{2}$ of SFP (5.1) is nonempty and the following conditions are satisfied:

(i) $\alpha_{n}+\beta_{n}+\gamma_{n}=1$, for each $n \geq 0$;

(ii) $\lim _{n \rightarrow \infty} \beta_{n}=0$, and $\sum_{n=0}^{\infty} \beta_{n}=\infty$;

(iii) $\liminf _{n \rightarrow \infty} \alpha_{n} \gamma_{n}>0$;

(iv) $\left\{\lambda_{n}\right\} \subset\left(0, \frac{2}{L}\right)$, where $L=\|U\|^{2}$,

then the sequence $\left\{w_{n}\right\}$ defined by (5.2) converges strongly to a solution $w^{*}$ of SFP (5.1) and $w^{*}=P_{\Gamma_{2}} f\left(w^{*}\right)$. 
Proof In Theorem 4.2 taking $H_{2}=H_{3}, B=I, G=U,\left\{C_{i}\right\}=\{C\}$, and $\left\{Q_{i}\right\}=\{Q\}$, the conclusions of Theorem 5.1 can be obtained from Theorem 4.2 immediately.

Remark Theorem 5.1 generalizes and extends the main results of Censor and Elfving [1] and Censor and Segal [19] from weak convergence to strong convergence.

\subsection{Application to null point problem of maximal monotone operators}

Let $H_{1}, H_{2}, H_{3}, A, B$, be the same as in Theorem 3.3. Let $M: H_{1} \rightarrow H_{1}$, and $N: H_{2} \rightarrow H_{2}$ be two strictly maximal monotone operators. It is well known that the associated resolvent mappings $J_{\mu}^{M}(x):=(I+\mu M)^{-1}$ and $J_{\mu}^{N}(x):=(I+\mu N)^{-1}$ of $M$ and $N$, respectively, are oneto-one nonexpansive mappings, and

$$
x \in M^{-1}(0) \Leftrightarrow x \in F\left(J_{\mu}^{M}\right) ; \quad y \in N^{-1}(0) \quad \Leftrightarrow \quad y \in F\left(J_{\mu}^{N}\right) .
$$

Denote $S=J_{\mu}^{M}, T=J_{\mu}^{N}, C=M^{-1}(0)=F\left(J_{\mu}^{M}\right)$, and $Q=N^{-1}(0)=F\left(J_{\mu}^{N}\right)$, then the general split equality fixed point problem (1.7) is reduced to the following null point problem related to strictly maximal monotone operators $M$ and $N(\mathrm{NPP}(M, N))$ :

$$
\text { to find } x^{*} \in M^{-1}(0), y^{*} \in N^{-1}(0) \text { such that } A x^{*}=B y^{*} \text {. }
$$

From Theorem 3.3 we can obtain the following.

Theorem 5.2 Let $H_{1}, H_{2}, H_{3}, A, B, f, G$, be the same as in Theorem 3.3. Let $C, Q, S$, and $T$ be the same as above. Let $\left\{w_{n}\right\}$ be the sequence generated by $w_{0} \in H_{1} \times H_{2}$

$$
w_{n+1}=P\left[\alpha_{n} w_{n}+\beta_{n} f\left(w_{n}\right)+\gamma_{n} K\left(I-\lambda_{n} G^{*} G\right) w_{n}\right], \quad n \geq 0,
$$

where $P=\left[\begin{array}{l}P_{C} \\ P_{Q}\end{array}\right], K=\left[\begin{array}{l}S \\ T\end{array}\right]$. If the solution set $\Gamma_{3}$ of $\mathrm{NPP}(M, N)(5.5)$ is nonempty and the following conditions are satisfied:

(i) $\alpha_{n}+\beta_{n}+\gamma_{n}=1$, for each $n \geq 0$;

(ii) $\lim _{n \rightarrow \infty} \beta_{n}=0$, and $\sum_{n=0}^{\infty} \beta_{n}=\infty$;

(iii) $\liminf _{n \rightarrow \infty} \alpha_{n} \gamma_{n}>0$;

(iv) $\left\{\lambda_{n}\right\} \subset\left(0, \frac{2}{L}\right)$, where $L=\|G\|^{2}$,

then the sequence $\left\{w_{n}\right\}$ defined by (5.6) converges strongly to $w^{*}=P_{\Gamma_{3}} f\left(w^{*}\right)$, which is a solution of $\mathrm{NPP}(M, N)(5.5)$.

Proof Since $S=J_{\mu}^{M}$ and $T=J_{\mu}^{N}$ both are one-to-one nonexpansive with $F(S) \neq \varnothing$ and $F(T) \neq \emptyset$. Hence they are one-to-one quasi-nonexpansive mappings and $I-K\left(I-\lambda_{n} G^{*} G\right)$ is demi-closed at zero. Therefore all conditions in Theorem 3.3 are satisfied. The conclusions of Theorem 5.2 can be obtained from Theorem 3.3 immediately.

\subsection{Application to equality equilibrium problem}

Let $D$ be a nonempty closed and convex subset of a real Hilbert $H$. A bifunction $g: D \times$ $D \rightarrow(-\infty,+\infty)$ is said to be a equilibrium function, if it satisfies the following conditions:

(A1) $g(x, x)=0$, for all $x \in D$;

(A2) $g$ is monotone, i.e., $g(x, y)+g(y, x) \leq 0$ for all $x, y \in D$;

(A3) $\lim \sup _{t \downarrow 0} g(t z+(1-t) x, y) \leq g(x, y)$ for all $x, y, z \in D$; 
(A4) for each $x \in D, y \mapsto g(x, y)$ is convex and lower semi-continuous.

The so-called equilibrium problem with respective to the equilibrium functions $g$ and $D$ is

to find $x^{*} \in D$ such that $g\left(x^{*}, y\right) \geq 0, \quad \forall y \in D$.

Its solution set is denoted by $\operatorname{EP}(g, D)$.

For given $\lambda>0$ and $x \in H$, the resolvent of the equilibrium function $g$ is the operator $R_{\lambda, g}: H \rightarrow D$ defined by

$$
R_{\lambda, g}(x):=\left\{z \in D: g(z, y)+\frac{1}{\lambda}\langle y-z, z-x\rangle \geq 0, \forall y \in D\right\} .
$$

It is well known that the resolvent $R_{\lambda, g}$ of the equilibrium function $g$ has the following properties [20]:

(1) $R_{\lambda, g}$ is single-valued;

(2) $F\left(R_{\lambda, g}\right)=\mathrm{EP}(g, D)$ and $F\left(R_{\lambda, g}\right)$ is a nonempty closed and convex subset of $D$;

(3) $R_{\lambda, g}$ is a nonexpansive mapping, and so it is quasi-nonexpansive.

Definition 5.3 Let $h, j: D \times D \rightarrow(-\infty,+\infty)$ be two equilibrium functions and, for given $\lambda>0$, let $R_{\lambda, h}$ and $R_{\lambda, j}$ be the resolvents of $h$ and $j$ (defined by (5.8)), respectively. Denote by $S=R_{\lambda, h}, T=R_{\lambda, j}, C:=F\left(R_{\lambda, h}\right)$, and $Q:=F\left(R_{\lambda, j}\right)$. Then the equality equilibrium problem with respective to the equilibrium functions $h, j$, and $D$ is

$$
\begin{gathered}
(\operatorname{EEP}(h, j, D)) \quad \text { to find } x^{*} \in F\left(R_{\lambda, h}\right), y^{*} \in F\left(R_{\lambda, j}\right) \text { such that } h\left(x^{*}, u\right) \geq 0, \\
\forall u \in D, j\left(y^{*}, v\right) \geq 0, \forall v \in D \text { and } A x^{*}=B y^{*},
\end{gathered}
$$

where $A, B: H \rightarrow H$ are two linear and bounded operators.

The following theorem can be obtained from Theorem 3.3 immediately.

Theorem 5.4 Let $H$ be a real Hilbert space, $D$ be a nonempty and closed convex subset of $H$. Let $G, f$ be the same as in Theorem 3.3. For given $\lambda>0$, let $h, j, R_{\lambda, h}, R_{\lambda, j}, S, T, C, Q$ be the same as above. Let $\left\{w_{n}\right\}$ be the sequence generated by $w_{0} \in H \times H$ :

$$
w_{n+1}=P\left[\alpha_{n} w_{n}+\beta_{n} f\left(w_{n}\right)+\gamma_{n} K\left(I-\lambda_{n} G^{*} G\right) w_{n}\right], \quad n \geq 0,
$$

where $P=\left[\begin{array}{l}P_{C} \\ P_{Q}\end{array}\right], K=\left[\begin{array}{l}S \\ T\end{array}\right]$. If the solution set $\Gamma_{4}$ of $\operatorname{EEP}(h, j, D)(5.9)$ is nonempty and the following conditions are satisfied:

(i) $\alpha_{n}+\beta_{n}+\gamma_{n}=1$, for each $n \geq 0$;

(ii) $\lim _{n \rightarrow \infty} \beta_{n}=0$, and $\sum_{n=0}^{\infty} \beta_{n}=\infty$;

(iii) $\liminf _{n \rightarrow \infty} \alpha_{n} \gamma_{n}>0$;

(iv) $\left\{\lambda_{n}\right\} \subset\left(0, \frac{2}{L}\right)$, where $L=\|G\|^{2}$,

then the sequence $\left\{w_{n}\right\}$ converges strongly to $w^{*}=P_{\Gamma_{4}} f\left(w^{*}\right)$, which is a solution of $\operatorname{EEP}(h$, $j, D)(5.9)$. 
Authors' contributions

All authors contributed equally to the writing of this paper. All authors read and approved the final manuscript.

\section{Author details}

${ }^{1}$ College of Statistics and Mathematics, Yunnan University of Finance and Economics, Kunming, Yunnan 650221, P.R. China. ${ }^{2}$ Department of Mathematics, Texas A\&M University-Kingsville, 700 University Blvd., Kingsville, TX 78363-8202, USA.

\section{Received: 14 May 2014 Accepted: 12 September 2014 Published: 25 Sep 2014}

\section{References}

1. Censor, Y, Elfving, T: A multiprojection algorithm using Bregman projections in a product space. Numer. Algorithms 8 , 221-239 (1994)

2. Byrne, C: Iterative oblique projection onto convex subsets and the split feasibility problem. Inverse Probl. 18, 441-453 (2002)

3. Censor, Y, Bortfeld, T, Martin, N, Trofimov, A: A unified approach for inversion problem in intensity-modulated radiation therapy. Phys. Med. Biol. 51, 2353-2365 (2006)

4. Censor, Y, Elfving, T, Kopf, N, Bortfeld, T: The multiple-sets split feasibility problem and its applications. Inverse Probl. 21, 2071-2084 (2005)

5. Censor, Y, Motova, A, Segal, A: Perturbed projections and subgradient projections for the multiple-sets split feasibility problem. J. Math. Anal. Appl. 327, 1244-1256 (2007)

6. Xu, HK: A variable Krasnosel'skii-Mann algorithm and the multiple-sets split feasibility problem. Inverse Probl. 22, 2021-2034 (2006)

7. Yang, Q: The relaxed CQ algorithm for solving the split feasibility problem. Inverse Probl. 20, 1261-1266 (2004)

8. Zhao, J, Yang, Q: Several solution methods for the split feasibility problem. Inverse Probl. 21, 1791-1799 (2005)

9. Chang, SS, Cho, YJ, Kim, JK, Zhang, WB, Yang, L: Multiple-set split feasibility problems for asymptotically strict pseudocontractions. Abstr. Appl. Anal. 2012, Article ID 491760 (2012). doi:10.1155/2012/491760

10. Chang, SS, Wang, L, Tang, YK, Yang, L: The split common fixed point problem for total asymptotically strictly pseudocontractive mappings. J. Appl. Math. 2012, Article ID 385638 (2012). doi:10.1155/2012/385638

11. Moudafi, A: A relaxed alternating CQ-algorithm for convex feasibility problems. Nonlinear Anal. 79, 117-121 (2013)

12. Moudafi, A, Al-Shemas, E: Simultaneous iterative methods for split equality problem. Trans. Math. Program. Appl. 1, 1-11 (2013)

13. Moudafi, A: Alternating CQ-algorithm for convex feasibility and split fixed-point problems. J. Nonlinear Convex Anal. (2012)

14. Eslamian, M, Latif, A: General split feasibility problems in Hilbert spaces. Abstr. Appl. Anal. 2013, Article ID 805104 (2013)

15. Chen, RD, Wang, J, Zhang, HW: General split equality problems in Hilbert spaces. Fixed Point Theory Appl. 2014,35 (2014)

16. Chang, SS, Lee, HWJ, Chan, CK, Zhang, WB: A modified Halpern-type iterative algorithm for totally quasi-asymptotically nonexpansive mappings with applications. Appl. Math. Comput. 218, 6489-6497 (2012)

17. Chang, SS: On Chidume's open questions and approximate solutions for multi-valued strongly accretive mapping equations in Banach spaces. J. Math. Anal. Appl. 216, 94-111 (1997)

18. Maingé, P-E: Strong convergence of projected subgradient methods for nonsmooth and nonstrictly convex minimization. Set-Valued Anal. 16(7-8), 899-912 (2008)

19. Censor, Y, Segal, A: The split common fixed point problem for directed operators. J. Convex Anal. 16, 587-600 (2009)

20. Blum, E, Oettli, W: From optimization and variational inequalities to equilibrium problems. Math. Stud. 63(1/4), 123-145 (1994)

10.1186/1029-242X-2014-367

Cite this article as: Chang and Agarwal: Strong convergence theorems of general split equality problems for quasi-nonexpansive mappings. Journal of Inequalities and Applications 2014, 2014:367

\section{Submit your manuscript to a SpringerOpen ${ }^{\circ}$ journal and benefit from:}

- Convenient online submission

Rigorous peer review

- Immediate publication on acceptance

- Open access: articles freely available online

- High visibility within the field

- Retaining the copyright to your article 